age of onset of seizures, seizure frequency, seizure type, and use of AEDs with cognitive side effects. ADHD-C and epilepsy comorbidity is a marker for severe epilepsy and/or severe brain dysfunction. Characteristics of ADHD/epilepsy comorbid cases differ from those of 'primary' ADHD in the prominence of inattentiveness as a core symptom. Impulsivity and dyscontrol are more frequent as core symptoms in children with primary ADHD (Barkley, 2006).

For a comprehensive review of ADHD and epilepsy, supported by 108 references, see Schubert R, 2005, included in Ped Neur Briefs Jan 2005;19:1-2; All types of epilepsy and even children with recent onset of seizures are involved. Patients with absence epilepsy have difficulty sustaining attention despite adequate seizure control and normal IQ. Other forms of epilepsy frequently complicated by ADHD include electrical status epilepticus during slowwave sleep, and benign rolandic epilepsy. Brief cognitive deficits (transient cognitive impairment) occur with subclinical epileptiform discharges, and may adversely affect attention and cognitive function without the occurrence of clinical seizures. AED use in these patients is controversial. Treatment of ADHD with methylphenidate (MPH) is effective and safe in patients with epilepsy, provided seizures are controlled with AEDs. (Feldman H et al. Am J Dis Child 1989;143:1081-1086; Tan M et al. Arch Dis Child 2005;90:57-59). MPHtreatment of ADHD complicated by centrotemporal (rolandic) spikes in the EEG has a risk of precipitating seizures in $16.7 \%$ compared to only $0.6 \%$ in patients with normal EEGs. (Hemmer SA et al. Pediatr Neurol 2001;24:99-102).

\title{
ATTENTION AND NEUROCOGNITIVE IMPAIRMENT IN ADHD
}

Specific executive functions (EFs) and attention deficit patterns in ADHD subtypes were studied in 50 boys (ages 8-14 years, mean 10.42) with ADHD and 44 controls, at the University of Rome, Italy. The executive functions test battery included the Wisconsin Card Sorting Test (measure of cognitive flexibility), Stroop Test (interference control), Tower of London (planning ability), Digit Span Backwards (verbal working memory), FAS Test (verbal fluency), Trail Making Test (visual search and divided attention), Continuous Performance Test II (sustained attention and inhibition), and visual-spatial, visual-object, and phonological working memory tests. ADHD patients, both inattentive and combined subtypes, differed from controls on tests of response inhibition, divided attention, phonological, and visual object working memory, and reaction times. Executive functioning profiles were similar for ADHD subtypes. Response inhibition predicts performance on working memory tests but not on divided attention/set shifting and on sustained attention. Boys with ADHD have a selective impairment of executive functions and attention tasks. Neural circuits that control response inhibition (right prefrontal cortex) and divided attention (left dorsolateral prefrontal cortex) in ADHD subtypes are involved independently in the pathogenesis of neuropsychological deficits. Impairments in phonological and visual-object working memory are additional characteristics of ADHD males with inattentive or combined subtypes. (Pasini A, Paloscia C, Alessandrelli R, Porfirio MC, Curatolo P. Attention and executive functions profile in drug naïve ADHD subtypes. Brain Dev August 2007;29:400408). (Respond: Dr Augusto Pasini, Department of Neuroscience, University of Rome "Tor Vergata", Via Alberico 2 n.35, 00193 Rome, Italy). 
COMMENT. Executive functions may be defined as mental control cognitive processes that facilitate goal directed behavior. They include response inhibition, planning, cognitive flexibility/set shifting, working memory, and verbal fluency. Attention is defined as a set of processes that enhance cognitive, motor, and sensory processing. Sustained attention is the ability to maintain performance without distraction during continuous activities. Divided attention is the ability to simultaneously respond to multiple stimuli. The present study investigated all five executive functioning domains and attention in ADHD subtypes. The ADHD subtypes (inattentive and combined) are homogeneous in neuropsychological profiles except for the role of interference inhibition on working memory. Inhibition of interference predicts performance on working memory only in the inattentive subtype. The findings support the involvement of partially independent neuronal circuits that control inhibition and divided attention in ADHD. Right prefrontal cortex controls response inhibition, while left dorsolateral prefrontal cortex modulates divided attention.

Role of neuropsychological tests in diagnosis of ADHD. In a study performed in Columbia and at the University of Georgia, neuropsychological measures, including the continuous performance tests (CPT), had strong sensitivity but weak specificity when used in diagnosis of ADHD. (Pineda DA et al. Pediatr Neurol 2007;36:373-381). CPT is not recommended as a measure for clinical diagnosis of ADHD. CPT is sensitive to attention problems caused by medication, depression, ADHD and other causes, and is not specific for ADHD. Neuropsychological measures are important in planning interventions for the ADHD child with concomitant learning problems or academic underachievement.

\section{METHYLPHENIDATE IN TREATMENT OF ADHD AND COMORBID CHRONIC TIC DISORDER}

The safety and efficacy of immediate-release methylphenidate (MPH-IR) for the treatment of attention deficit hyperactivity disorder (ADHD) in children (ages 6-12 years) with Tourette's syndrome $(96 \%)$ or chronic motor tic disorder $(4 \%)$ were evaluated at State University of New York, Stony Brook. Seventy one children, in 2 cohorts, were treated with three doses of MPH $(0.1,0.3$, and $0.5 \mathrm{mg} / \mathrm{kg})$ and placebo twice daily for 2 weeks each, double-blind, and effects were assessed with a battery of parent, teacher, child, and physician-completed rating scales and laboratory tasks. MPH-IR effectively suppressed ADHD, oppositional defiant disorder, and peer aggression behaviors, without altering the overall severity of tic disorder or obsessive compulsive disorder. Dose-response was variable. Teacher ratings indicated that MPH-IR treatment decreased the frequency and severity of the tic disorder. The physician's 2-minute Tic/Habit Count showed an increase in simple but not complex motor movements $(0.3$ and $0.5 \mathrm{mg} / \mathrm{kg}>$ placebo). For the Simulated Classroom, the tic frequency was slightly higher for the $0.5 \mathrm{mg} / \mathrm{kg}$ dose. Adverse effects related to dose included heart rate and blood pressure increases and weight loss. MPH-IR is not contraindicated as a short-term therapy for children with ADHD and chronic tic disorder, but treatment should be carefully monitored to exclude possible tic exacerbation in susceptible individuals. (Gadow KD, Sverd J, Nolan EE, Sprafkin J, Schneider J. Immediaterelease methylphenidate for ADHD in children with comorbid chronic multiple tic disorder. J Am Acad Child Adolesc Psychiatry July 2007;46:840-848). (Respond: Dr Kenneth 